\title{
Comparison of the Duo of Insulin-Like Growth Factor Binding Protein-1/Alpha Fetoprotein (Amnioquick Duo+®) and Nitrazine Test for Diagnosing Query Rupture of Fetal Membranes
}

\author{
Sherif Fekry Abd Allah Hendawy*, Sherif Hanafi Hussain*, **Jamila Ahmed Elmabrouk \\ * Department of Obstetrics and Gynaecology, Faculty of Medicine - Ain Shams University, ** Tripoli University \\ Corresponding author: Jamila Ahmed Elmabrouk, Mobile: 002010666866572, E-Mail: tantawy_wael@yahoo.com
}

\begin{abstract}
Background: premature rupture of membranes (PROM) constitutes one of the most important dilemmas in the obstetric practice. It could be defined as rupture of membranes before the onset of labour, irrespective of the gestational age. It is associated with infectious morbidity in mother and fetus, cord accidents, imminent term or preterm labour. For these reasons, its correct diagnosis is very important. A novel test used for diagnosing rupture of membranes (ROM) is Amnioquick duo+® (Biosynex, Strasbourg Cedex, France). Amnioquick duo $+\AA$ is a rapid strip test with immunoassay that is simple, easy to perform, quick and noninvasive. It is an immunochromatographic test that identifies even trace amounts of both alpha fetoprotein (AFP) and insulinlike growth factor binding protein-1 (IGFBP-1). On other hand the traditional methods for diagnosis of PROM are through the patient's history, leakage, ferning test and nitrazine test. Aim of the Work: the aim of this study is to compare the accuracy of the duo of insulin-like growth factor binding protein-1/alpha fetoprotein (Amnioquick duo+ ${ }^{\circledR}$ ) and nitrazine test for diagnosing query rupture of fetal membranes in pregnant women with query PROM. Patients and Methods: this comparative cross sectional study was carried out at Ain Shams University Maternity Hospital between November 2017 to June 2018. Results: a total of one hundred and thirteen pregnant women $>24$ weeks of gestation were included in the study with age ranging between 18 and 42 years (mean \pm SD, $27 \pm 6$ years). Thirty eight (33.6\%) women were primigravidae and $75(66.4 \%)$ were multigravidae. Forty (35.4\%) women were primiparas while $63(64.6 \%)$ were multiparas. Twenty five $(22.1 \%)$ women experienced one or more previous abortion. The Amnioquick duo+ ${ }^{\circledR}$ test was more specific and accuracy in diagnosing rupture of membranes than Nitrazine test. The sensitivity and the specificity of Amnioquick duo+® test in diagnosing PROM was $100 \%$ and $97.3 \%$ respectively as compared to Nitrazine test which was $100 \%$ and $83 \%$. The PPV and NPV of Amnioquick duo+ ${ }^{\circledR}$ test were $98.7 \%$ and $100 \%$ as compared to Nitrazine test which were $92.7 \%$ and $100 \%$. Conclusion: this study concluded that IGFBP-1/AFP (AmnioQuick ${ }^{\circledR}$ Duo+) test is rapid reliable non-invasive, easy and accurate bedside immunoassay test, better than nitrazine test and can used as complementary test to improve the management of women with women premature fetal membranes rupture.
\end{abstract}

Keywords: Amnioquick duo+®, Nitrazine, PROM, IGFBP-1/AFP

\section{INTRODUCTION}

Premature (prelabor) rupture of membranes (PROM) is defined as rupture of the fetal membranes spontaneously prior to the onset of uterine contractions ${ }^{(\mathbf{1})}$. It is a relatively frequent obstetric phenomenon occurring in $2-18 \%$ of pregnancies ${ }^{(2)}$. When it is preterm, it is often associated with prematurity-related complications including premature birth, pulmonary hypoplasia, fetal deformities and infectious materno-fetal morbidity ${ }^{(3)}$. The diagnosis of PROM is straight forward in the presence of obvious rupture of membranes. However, several factors such as urine, vaginal discharge or semen may interfere with traditional clinical assessment (TCA), leading to high levels of false negative and positive results. Such results may lead to inappropriate interventions such as hospitalization and stimulation of labor ${ }^{(3)}$. On the other hand, misdiagnosis of PROM may cause providers to withhold appropriate therapy ${ }^{(\mathbf{4})}$. A novel test used for diagnosing rupture of membranes (ROM) is Amnioquick duo+ ${ }^{\circledR}$ (Biosynex, Strasbourg Cedex, France). Amnioquick duo+ ${ }^{\circledR}$ is a rapid strip test with immunoassay that is simple, easy to perform, quick and noninvasive. It is an immunochromatographic test that identifies even trace amounts of both alpha fetoprotein (AFP) and insulin-like growth factor binding protein-1 (IGFBP-1). The protein markers, though abundant in amniotic fluid, are present in far lower concentrations or undetectable in the maternal blood or in cervicovaginal secretions in the genuine absence of rupture of membranes ${ }^{(5)}$. Thus, such a differential concentration between amniotic fluid and cervicovaginal secretions of the biomarkers makes Amnioquick duo+ ${ }^{\circledR}$ an excellent marker for PROM. The test can be performed even when fluid is not obvious in the vagina and its execution does not require laboratory specialized equipment or specially trained personnel ${ }^{(3,5)}$. On other hand the traditional methods for diagnosis of PROM are through 
the patient's history, leakage, ferning test and nitrazine test. The histories told by the patients are sometimes subjective, and the statements may not be elaborate. The obvious leakage of amniotic fluids from the cervical os can indicate PROM, but we cannot always identify enough fluid to confirm, and sometimes there is no visual leakage in the vagina. The ferning test and the nitrazine test are two generally used methods for diagnosis of PROM. The ferning test has been associated with false positive results in $5-30 \%$ of patients and false negative results in $5-12.9 \%$ of patients. The nitrazine test is easily contaminated by other fluids, such as semen, urine, blood, and antiseptic solution. PROM is an important dilemma in clinical practice, and we urgently require alternative, accurate and fast methods to help us solve the problem ${ }^{(\boldsymbol{)}}$.

\section{AIM OF THE WORK}

The aim of this study is to compare the accuracy of the duo of insulin-like growth factor binding protein-1/alpha fetoprotein (Amnioquick duo+ ${ }^{\circledR}$ ) and nitrazine test for diagnosing query rupture of fetal membranes in pregnant women with query PROM.

\section{PATIENTS AND METHODS}

- Type of Study: Evaluation of the accuracy of diagnostic test, cross sectional.

- Study Setting: Ain Shams university maternity hospitals.

- Study Period: The study was started at November 2017 to June 2018.

\section{- Study Population}

Inclusion Criteria: 1. Pregnancy duration of 24 weeks or more. 2. Consenting pregnant women with symptoms, signs or complaints suggestive of membrane rupture. Exclusion Criteria: 1. Women with vaginal bleeding. 2. Uterine contractions. 3. Fetal anomalies. 4. Placental pathology that could cause oligohydramnios including intrauterine growth restriction. Sample size justification: The required sample size has been calculated using the Power Analysis and Sample Size 2008 software version 08.0.15 (PASS@ 2008, NCSS, LLC, Keysville, Utah, USA). A previous study reported that the nitrazine test had a sensitivity of $90.1 \%$ and a specificity of $69 \%$ for diagnosis of PROM. In contrast, the sensitivity and specificity of Amnioquick duo+ ${ }^{\circledR}$ were $97.6 \%$ and $97.9 \%$, respectively. That study reported that approximately $73 \%$ of women presenting with symptoms suspicious of PROM proved to actually have $\mathrm{PROM}^{(7)}$. So, it is calculated that a sample size of
96 women suspected of PROM would include 70 (73\%) patients who actually have PROM. This sample achieves $83 \%$ power (type 2 error $=0.17$ ) to detect a difference of $8 \%$ between the sensitivities of the 2 tests (98\% versus $90 \%$ ) and a power of $100 \%$ to detect a difference of $29 \%$ between the specificities of the 2 (98\% versus 69\%) tests using a two-sided binomial test with type I error of 0.05 (i.e., confidence of 95\%). Ethical considerations: The study was approved by the Ethics Board of Ain Shams University and an informed written consent was taken from each participant in the study.

Study procedures: History taking: Personal history: it includes name, age, occupation, marriage and special habits. Obstetric history: it includes parity, last menstrual period, expected date of delivery, gestational age, previous preterm labor and vaginal bleeding. Medical history: it includes diabetes mellitus, hypertension and hepatic or renal disease. Surgical history: it includes history of previous pelvic or abdominal surgeries. Examination: General examination: it includes blood pressure, arterial pulse and temperature. Abdominal examination: it includes fundal level, fundal grip, pelvic grip, uterine contractions, tenderness and scars of previous operations. Local examination: all women were putted in dorsal lithotomy position, using a proper light source and sterile gloves; sterile speculum free of gel will placed into vagina.

The Amnioquick duo+ immunoassay (Manufacturer company: Bio-synex) was performed according to the manufacturer's instructions (Biosynex, 12 rue Ettore Bugatti-CS 28006 67038, Strasbourg Cedex, France). By using Amnioquick duo+ ${ }^{\circledR}$, a sample of vaginal secretion was collected using a vaginal swab entwined with Sterile Nylon ${ }^{\circledR}$ positioned in the posterior fornix or in the cervical canal for $60 \mathrm{~s}$. Subsequently, the swab was either bathed in a buffer tube for $10 \mathrm{~s}$ or the swab tip broken off into the vial buffer and the mixtures shaken together. Three drops of the resultant liquid content were dropped in the specimen well on the cassette (supplied by the manufacturers) stick containing monoclonal antibodies to AFP and IGFBP-I, which absorbs the extracted specimen. The pink line appeared on the $\mathrm{A}$ and $\mathrm{B}$ zones on the cassette, respectively when the amniotic fluid contained AFP and IGFBP-1. The result was interpreted within $10 \mathrm{~min}$. There were three distinct zones on the cassette for AFP (A), IGFBP-1(B) and control $(\mathrm{C})$. The interpretation of the results was 
based on the manufacturer's predetermined criteria, classified as positive, negative or doubtful for ROM. The test was positive when the $\mathrm{C}$ and the $\mathrm{B}$ lines were both present or when the $\mathrm{A}$ and the $\mathrm{C}$ lines were both present for cases with gestational age $\geq 24$ weeks. The test was negative when both the $\mathrm{A}$ and the $\mathrm{B}$ lines were absent. Lines were evaluated as positive if a continuous line was observable, even if faint ${ }^{(6)}$.

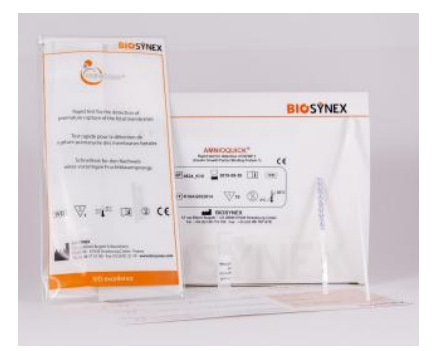

Fig. (1): Amnioquick duo+ immunoassay.

Nitrazine test (McolorPhast ${ }^{\mathrm{TH}}$ ): The $\mathrm{pH}$ test strip was inserted into the posterior vaginal fornix for about 30s, then removed from the vagina. A blue strip indicated PROM and other colors indicated no PROM.

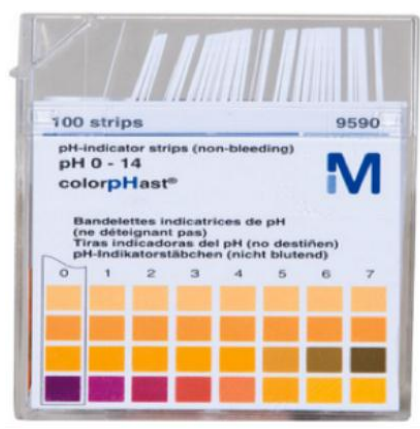

Fig. (2): Nitrazine test.

Statistical Methods: Data were analyzed using SPSS@ Statistics version 17 (SPSS@ Corp., Armonk, NY, USA) and XLSTAT(C) Version 2016.02.28451 (Addinsoft $\odot$, Paris, France). Continuous numerical variables were presented as mean $\pm \mathrm{SD}$ with range and categorical data as number and percentage. Diagnostic accuracy of the nitrazine test or AmnioQuick Duo+ ${ }^{\circledR}$ test was examined by contrasting the test result versus that of speculum examination as the gold-standard for diagnosis. The following diagnostic indices were calculated: sensitivity and specificity, positive and negative predictive value, positive and negative likelihood ratio, and overall accuracy. Inter-method agreement between the nitrazine test and AmnioQuick Duo $+\AA$ test was examined using Cohen's kappa coefficient $(\kappa)$, Scott's bias-adjusted kappa coefficient (BAK) and Bennet's prevalence adjusted and biasadjusted kappa coefficient (PABAK). The kappa coefficients are interpreted as follows:

\begin{tabular}{|c|c|}
\hline Kappa coefficient & Strength of agreement \\
\hline-1.0 to 0.0 & No or poor agreement \\
\hline $0.0-0.2$ & Slight \\
\hline $0.21-0.40$ & Fair \\
\hline $0.41-0.60$ & Moderate \\
\hline $0.61-0.8$ & Substantial \\
\hline $0.81-1.00$ & Nearly perfect \\
\hline
\end{tabular}

The McNemar test was used to compare paired categorical data. P-value $<0.05$ was considered statistically significant.

\section{RESULTS}

In this study, one hundred thirteen cases were enrolled and tested for PROM through: Sterile Cusco speculum examination (Golden standard test) where 76 patients were positive for rupture of membranes and 37 were negative for rupture of membrane, Nirazine test, AmnioQuick Duo+ ${ }^{\circledR}$ test. In this analysis, the comparison between Nitrazine versus AmnioQuick Duo+ ${ }^{\circledR}$ test for PROM diagnosis judged by sterile Cusco speculum examination (golden standard for PROM).

Table 1. Characteristics of the study population

\begin{tabular}{|l|c|}
\hline \multicolumn{1}{|c|}{ Variable } & $\begin{array}{c}\text { Mean } \pm \text { SD ] } \\
\text { (minimum }- \text { maximum) } \\
\text { /n (\%) }\end{array}$ \\
\hline Age (years) & $27 \pm 6(18-42)$ \\
\hline Gestational age (weeks) & $35.0 \pm 4.3(23.3-40.6)$ \\
\hline Gravidity & $38(33.6 \%)$ \\
\hline$P G$ & $0(0.0 \%)$ \\
\hline$G 1$ & $24(21.2 \%)$ \\
\hline$G 2$ & $19(16.8 \%)$ \\
\hline G3 & $18(15.9 \%)$ \\
\hline G4 & $14(12.4 \%)$ \\
\hline G5 or higher & \\
\hline Parity & $40(35.4 \%)$ \\
\hline P0 & $27(23.9 \%)$ \\
\hline P1 & $22(19.5 \%)$ \\
\hline P2 & $16(14.2 \%)$ \\
\hline P3 & $7(6.2 \%)$ \\
\hline P4 & $1(0.9 \%)$ \\
\hline P5 or higher & \\
\hline Previous abortion & $88(77.9 \%)$ \\
\hline Nil & $16(14.2 \%)$ \\
\hline One abortion & $6(5.3 \%)$ \\
\hline Two abortions & $2(1.8 \%)$ \\
\hline Three abortions & $1(0.9 \%)$ \\
\hline Four or more abortions & \\
\hline Da are mat & \\
\hline
\end{tabular}

Data are mean \pm SD (minimum - maximum) or number $(\%)$.

Table 1 shows the characteristics of the study population. The age ranged between 18 and 42 years (mean $\pm \mathrm{SD}, 27 \pm 6$ years). 38 (33.6\%) women 
were primigravidae and 75 (66.4\%) were multigravidae. $40(35.4 \%)$ women were primiparas while 63 (64.6\%) were multiparas. $25(22.1 \%)$ women experienced one or more previous abortion.

Table 2. Results of the nitrazine test, AmnioQuick Duo+ ${ }^{\circledR}$ test and speculum examination

\begin{tabular}{|l|c|}
\hline \multicolumn{1}{|c|}{ Variable } & $\boldsymbol{n}(\%)$ \\
\hline Nitrazine test & \\
\hline Negative & $31(27.4 \%)$ \\
\hline Positive & $82(72.6 \%)$ \\
\hline AmnioQuick Duo+® test & \\
\hline Negative & $36(31.9 \%)$ \\
\hline Positive & $77(68.1 \%)$ \\
\hline Speculum examination & \\
\hline Negative & $37(32.7 \%)$ \\
\hline Positive & $76(67.3 \%)$ \\
\hline
\end{tabular}

Data are number (\%).

Table 2 shows the results of the nitrazine test, AmnioQuick Duo+ ${ }^{\circledR}$ test and speculum examination. Eighty two (72.6\%) patients were positive for ROM by the nitrazine test while only 77 (68.1\%) patients were positive by the AmnioQuick Duo+ ${ }^{\circledR}$ test. By speculum examination (goldstandard test), 37 (32.7\%) patients were negative and $76(67.3 \%)$ patients were positive for ROM.

Table 3. Accuracy of the nitrazine test examined versus speculum examination as the gold-standard for diagnosis of ROM

\begin{tabular}{|l|c|c|c|}
\hline & \multicolumn{3}{|c|}{ Speculum examination } \\
\hline Nitrazine test & Positive & Negative & Column Total \\
\hline Positive & 76 & 6 & 82 \\
\hline Negative & 0 & 31 & 31 \\
\hline Total & 76 & 37 & 113 \\
\hline Statistic & Value & Lower bound & Upper bound \\
\hline Correct classification & $94.7 \%$ & $90.6 \%$ & $98.8 \%$ \\
\hline Misclassification & $5.3 \%$ & $1.2 \%$ & $9.4 \%$ \\
\hline Sensitivity & $100.0 \%$ & $94.1 \%$ & $100.0 \%$ \\
\hline Specificity & $83.8 \%$ & $68.4 \%$ & $92.6 \%$ \\
\hline False positive rate & $16.2 \%$ & $4.9 \%$ & $27.5 \%$ \\
\hline False negative rate & $0.0 \%$ & $0.0 \%$ & $0.0 \%$ \\
\hline $\begin{array}{l}\text { Prevalence } \\
\text { Positive predictive } \\
\text { value (PV+) }\end{array}$ & $67.3 \%$ & $58.6 \%$ & $75.9 \%$ \\
\hline $\begin{array}{l}\text { Negative predictive } \\
\text { value (PV-) }\end{array}$ & $100.0 \%$ & $100.0 \%$ & $100.0 \%$ \\
\hline $\begin{array}{l}\text { Positive likelihood } \\
\text { ratio (LR+) }\end{array}$ & 6.17 & 2.96 & 12.83 \\
\hline $\begin{array}{l}\text { Negative likelihood } \\
\text { ratio (LR-) }\end{array}$ & 0.00 & $87.0 \%$ & $98.3 \%$ \\
\hline
\end{tabular}

Data are counts.

Table 3 shows a cross-tabulation of the results of the nitrazine test examined versus those of speculum examination as the gold-standard for diagnosis of ROM. Eighty two patients tested positive by the nitrazine test. Of these, 76 were truly positive and 6 were falsely positive by speculum examination. Thirty one patients were negative by the nitrazine test, all of whom were truly negative by speculum examination. These findings are illustrated in Figure 3. From these values, it is estimated that the nitrazine test has an accuracy (correct classification rate) of $94.7 \%$, sensitivity of $100 \%$, specificity of $83 \%$, positive predictive value $(\mathrm{PV}+)$ of $92.7 \%$, negative predictive value (PV-) of $100 \%$, Positive likelihood ratio (LR+) of 6.17 and negative likelihood ratio (LR-) of 0.0.

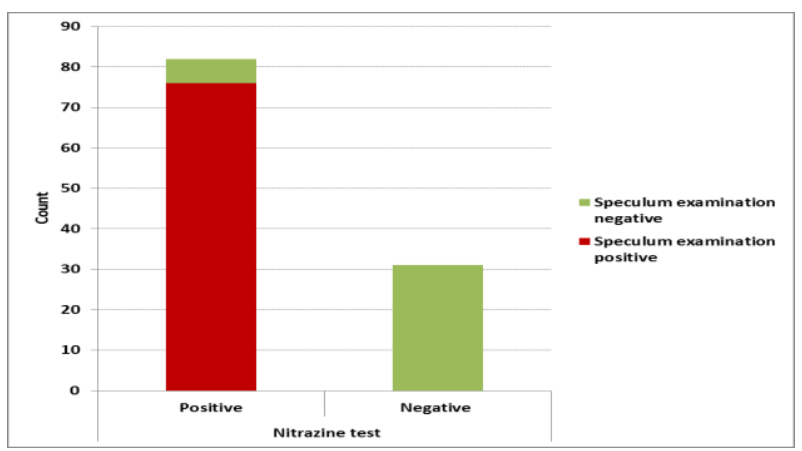

Figure 3. Results of the nitrazine test examined versus speculum examination as the gold-standard for diagnosis of ROM.

Table 4. Accuracy of the AmnioQuick Duo+® test examined versus speculum examination as the gold-standard for diagnosis of ROM

\begin{tabular}{|c|c|c|c|}
\hline & \multicolumn{3}{|c|}{ Speculum examination } \\
\hline $\begin{array}{l}\text { AmnioQuick } \\
\text { Duo+® test }\end{array}$ & Positive & Negative & Column Tota \\
\hline Positive & 76 & 1 & 77 \\
\hline Negative & 0 & 36 & 36 \\
\hline Total & 76 & 37 & 113 \\
\hline & & & $\% \mathrm{CI}$ \\
\hline Statistic & Value & Lower boun & Upper bound \\
\hline Correct classification & $99.1 \%$ & $97.4 \%$ & $100.0 \%$ \\
\hline Misclassification & $0.9 \%$ & $0.0 \%$ & $2.6 \%$ \\
\hline Sensitivity & $100.0 \%$ & $94.1 \%$ & $100.0 \%$ \\
\hline Specificity & $97.3 \%$ & $84.7 \%$ & $100.0 \%$ \\
\hline False positive rate & $2.7 \%$ & $0.0 \%$ & $7.7 \%$ \\
\hline False negative rate & $0.0 \%$ & $0.0 \%$ & $0.0 \%$ \\
\hline Prevalence & $67.3 \%$ & $58.6 \%$ & $75.9 \%$ \\
\hline $\begin{array}{l}\text { Positive predictive } \\
\text { value }(\mathrm{PV}+)\end{array}$ & $98.7 \%$ & $96.2 \%$ & $100.0 \%$ \\
\hline $\begin{array}{l}\text { Negative predictive } \\
\text { value (PV-) }\end{array}$ & $100.0 \%$ & $100.0 \%$ & $100.0 \%$ \\
\hline $\begin{array}{l}\text { Positive likelihood } \\
\text { ratio (LR+) }\end{array}$ & 37.00 & 5.35 & 255.75 \\
\hline $\begin{array}{l}\text { Negative likelihood } \\
\text { ratio (LR-) }\end{array}$ & 0.00 & & \\
\hline
\end{tabular}

Data are counts. 
Table 4 shows a cross-tabulation of the results of the AmnioQuick Duo+® test examined versus those of speculum examination as the goldstandard for diagnosis of ROM. 77 patients tested positive by the AmnioQuick Duo+® test. Of these, 76 were truly positive and only 1 was falsely positive by speculum examination. 36 patients were negative by the AmnioQuick Duo+® test, all of whom were truly negative by speculum examination. These findings are illustrated in Figure 4. From these values, it is estimated that the AmnioQuick Duo+® test has an accuracy (correct classification rate) of $99.1 \%$, sensitivity of $100 \%$, specificity of $97.3 \%$, positive predictive value $(\mathrm{PV}+)$ of $98.7 \%$, negative predictive value (PV-) of $100 \%$, Positive likelihood ratio (LR+) of 37.0 and negative likelihood ratio (LR-) of 0.0 . Figure 5 shows a summary of the diagnostic performance of the AmnioQuick Duo+ ${ }^{\circledR}$ and nitrazine tests.

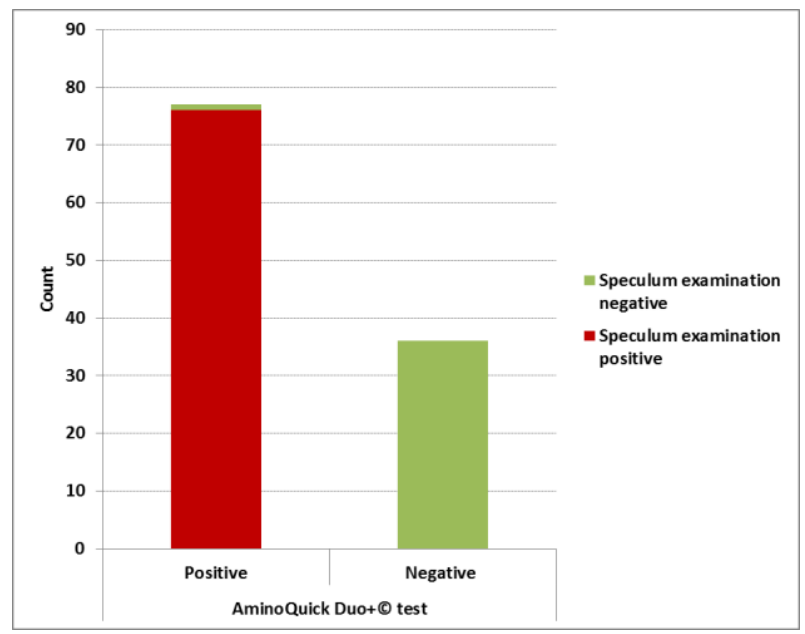

Figure 4. Results of the AmnioQuick Duo+ ${ }^{\circledR}$ test examined versus speculum examination as the goldstandard for diagnosis of ROM.

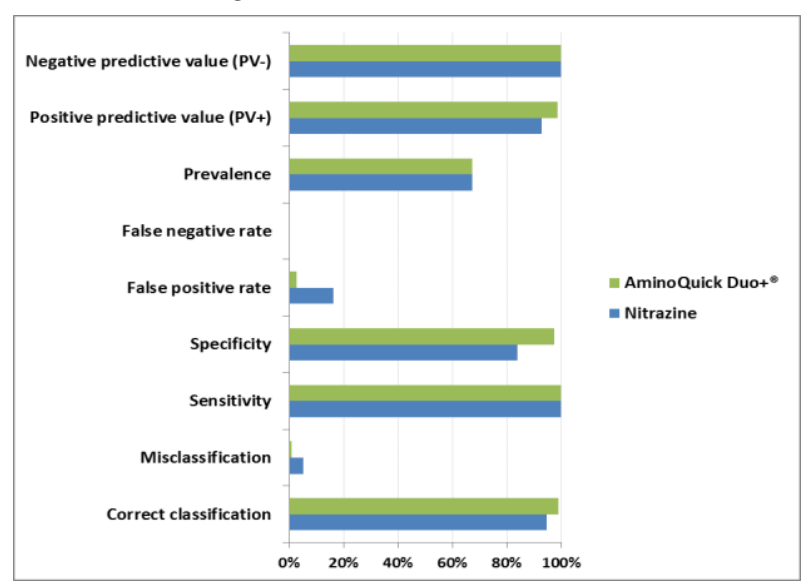

Figure 5. The diagnostic performance of the AmnioQuick Duo+ ${ }^{\circledR}$ and nitrazine tests.
Table 5. Agreement between the AmnioQuick Duo+ ${ }^{\circledR}$ test and nitrazine test

\begin{tabular}{|l|c|c|c|}
\hline & \multicolumn{3}{|c|}{ AmnioQuick Duo+® test } \\
\hline \multicolumn{1}{|c|}{ Nitrazine test } & Positive & Negative & $\begin{array}{c}\text { Column } \\
\text { Total }\end{array}$ \\
\hline Positive & 76 & 6 & 82 \\
\hline Negative & 1 & 30 & 31 \\
\hline Total & 77 & 36 & 113 \\
\hline Measures of agreement & & & \\
\hline Cohen's kappa & 0.85 & & \\
\hline Scott's kappa (BAK) & 0.85 & & \\
\hline Bennett's kappa (PABAK) & $0.88 *$ & & \\
\hline
\end{tabular}

Data are counts.

A Bennett's kappa (PABAK) of 0.88 denotes nearly perfect agreement between the AmnioQuick Duo+ ${ }^{\circledR}$ and nitrazine tests. Table 6 represents a cross-tabulation of the results of the AmnioQuick Duo+® test versus those of the nitrazine test. 76 patients tested positive and 30 patients tested negative by both tests. 1 patient tested positive by the AmnioQuick Duo+ ${ }^{\circledR}$ test and negative by the nitrazine test. Conversely, 6 patients tested negative by the AmnioQuick Duo+ ${ }^{\circledR}$ test and positive by the nitrazine test. These findings are illustrated in Figure 6. From these figures, the Bennett kappa coefficient (Prevalence-adjusted bias-adjusted kappa coefficient, PABAK) is estimated to be 0.88 which denotes nearly perfect agreement between both tests.

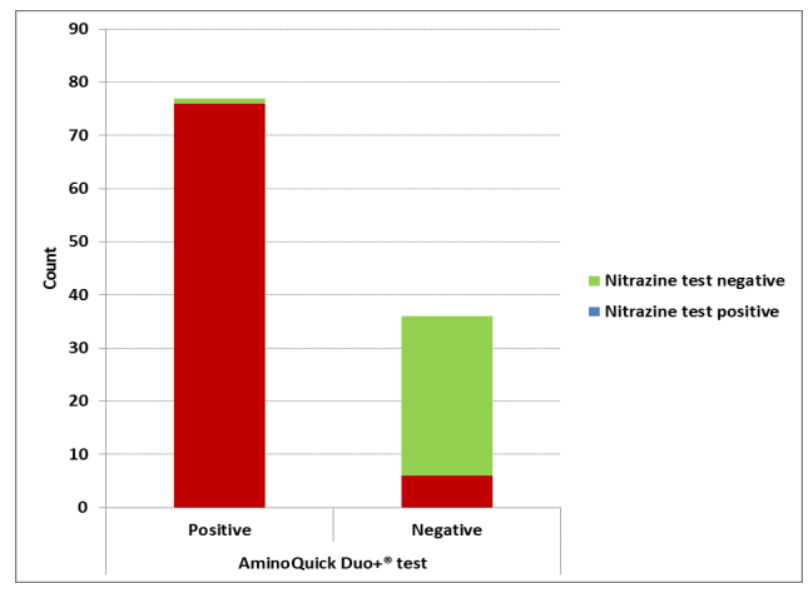

Figure 6. Results of the AmnioQuick Duo+ ${ }^{\circledR}$ test examined versus the nitrazine test. 
Table 6. Cross-tabulation of the results of the AmnioQuick Duo+ ${ }^{\circledR}$ test versus that of the nitrazine test in the subgroup of patients.

\begin{tabular}{|l|c|c|c|}
\hline & \multicolumn{3}{|c|}{ AmnioQuick Duo+® test } \\
\hline Nitrazine test & Negative & Positive & Column Total \\
\hline Negative & 30 & 1 & $31(83.8 \%)$ \\
\hline Positive & 6 & 0 & $6(16.2 \%)$ \\
\hline Total & $36(97.3 \%)$ & $1(2.7 \%)$ & 37 \\
\hline McNemar test & & & \\
\hline Difference & $13.51 \%$ & & \\
\hline 95\% CI & $0.19 \%$ to $26.84 \%$ & & \\
\hline p-value & 0.125 & & \\
\hline
\end{tabular}

Data are counts and percentages.

Table 6 shows a cross-tabulation of the results of the AmnioQuick Duo+ ${ }^{\circledR}$ test versus that of the nitrazine test in the subgroup of patients with negative speculum examination which is the goldstandard for diagnosis of ROM. Of the 37 patients who tested negative by speculum examination, 30 patients tested negative by both the AmnioQuick Duo+ ${ }^{\circledR}$ test and the nitrazine test. One patient tested positive by the AmnioQuick Duo+® test but was negative by the nitrazine test. In contrast, 6 patients tested negative by the AmnioQuick Duo+ ${ }^{\circledR}$ test but were negative by the nitrazine test. These findings are illustrated in Figure 7. To compare the specificities of both tests, the McNemar test was conducted on this paired data and showed no statistically significant difference in the percentage of patients testing negative by either test $(97.3 \%$ for the AmnioQuick Duo+ ${ }^{\circledR}$ test versus $83.8 \%$ versus the nitrazine test, difference $=13.51 \%, 95 \% \mathrm{CI}=$ $0.19 \%$ to $26.84 \%$, p-value $=0.125$ ) .

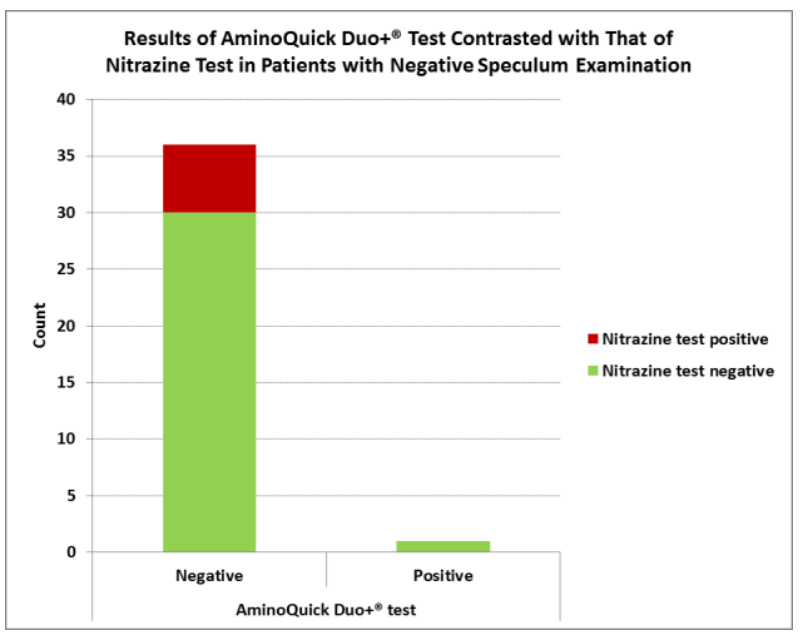

Figure 7. Results of the AmnioQuick Duo+ ${ }^{\circledR}$ test contrasted with that of the nitrazine test in the subgroup of patients with negative speculum examination.
Table 7. Cross-tabulation of the results of the AmnioQuick Duo+ ${ }^{\circledR}$ test versus that of the nitrazine test in the subgroup of patients.

\begin{tabular}{|l|c|c|c|}
\hline & \multicolumn{3}{|c|}{ AmnioQuick Duo+® test } \\
\hline Nitrazine test & Negative & Positive & Column Total \\
\hline Negative & 0 & 0 & $0(0 \%)$ \\
\hline Positive & 0 & 76 & $76(100 \%)$ \\
\hline Total & $0(0 \%)$ & $76(100 \%)$ & 76 \\
\hline McNemar test & & & \\
\hline Difference & $0 \%$ & & \\
\hline $95 \%$ CI & NA & & \\
\hline p-value & NA & & \\
\hline
\end{tabular}

Data are counts and percentages. NA=test not applicable.

Table 7 shows a cross-tabulation of the results of the AmnioQuick Duo+® test versus that of the nitrazine test in the subgroup of patients with positive speculum examination. Of the 76 patients who tested positive by speculum examination, all were positive by both the AmnioQuick Duo+® test and the nitrazine test. These findings are illustrated in Figure 8. The McNemar test could not be conducted on this paired data in order to compare the sensitivity of either test, since all patients $(\mathrm{n}=76)$ tested positive by both tests.

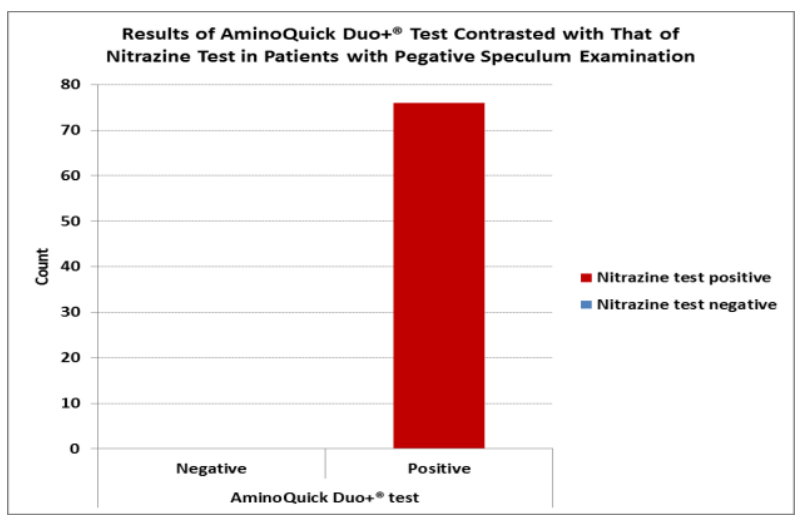

Figure 8. Results of the AmnioQuick Duo+ ${ }^{\circledR}$ test contrasted with that of the nitrazine test in the subgroup of patients with positive speculum examination.

\section{DISCUSSION}

Premature (prelabor) rupture of membranes (PROM) is defined as rupture of the fetal membranes spontaneously prior to the onset of uterine contractions ${ }^{(1)}$. PROM is preterm (PPROM) when it occurs before 37 weeks gestation ${ }^{(8)}$. It is a relatively frequent obstetric phenomenon occurring in $2-18 \%$ of pregnancies. When it is preterm, it is often associated with prematurity-related complications including 
premature birth, pulmonary hypoplasia, fetal deformities and infectious materno-fetal morbidity (9). Accurate diagnosis of PROM remains a frequent clinical problem in obstetrics. Unfortunately, a non-invasive standard diagnostic test is not available at this time. The diagnosis of PROM is usually based on the patient's history, identification of gross pooling of amniotic fluids from the cervical canal during sterile speculum examination, after microscopic examination and the Nitrazine test ${ }^{(10)}$. Despite the theoretic advantages of using Amnioquick duo+ ${ }^{\circledR}$ as a marker of ROM, there is still a deficiency of prospective data on the performance matrix of the immunoassay ${ }^{(5)}$. There are many new bedside biological tests that were developed to detect certain biochemical markers that are highly present in the amniotic fluid but not in the vaginal fluid. Some of the biochemical markers that are used to detect membranes leakage such as alphafetoprotein (AFP), insulin-like growth factorbinding protein-1 (IGFBP-1), placental alphamicroglobulin-1 and fibronectin are targeted ${ }^{(\mathbf{1 1})}$. The approach to the diagnosis of rupture of membranes is clinical, with over $90 \%$ of cases being confirmed based on the presence of a suspicious history or ultiasonographic finding followed by documentation of fluid passing from the cervix or the presence of a nitrazine/ ferning positive vaginal pool of fluid. Various methods are used to diagnose PROM such as nitrazin and ferning test but have low sensitivity and specificity, or injection of intra-amniotic dye, although are very reliable test but are invasive with serious complications (12). This study was trying to investigate a non-invasive, simple, rapid, easy and cheap method for diagnosis of premature rupture of membrane. We demonstrated that Amnioquick duo+ was superior to traditional clinical assessment (nitrazine test) for diagnosing premature rupture of fetal membranes (PROM) in pregnant women presenting with symptoms or signs of drainage of liquor. We found a diagnostic accuracy of $99.1 \%$ for Amnioquick duo+ versus $94.7 \%$ for TCA. The sensitivity and the specificity of Amnioquick duo+ test in diagnosing PROM was $100 \%$ and $97.3 \%$ respectively as compared to Nitrazine test which was $100 \%$ and $83 \%$. The PPV and NPV of Amnioquick duo+ test were $98.7 \%$ and $100 \%$ as compared to Nitrazine test which were $92.7 \%$ and $100 \%$. The sensitivity of the Amnioquick duo+ test was high in detecting ROM. The reason for this is because the IGFBP-1/AFP test can detect low levels of both IGFBP-1 (10 $\left.\mathrm{ng} \mathrm{ml}^{-1}\right)$ and AFP $\left(5 \mathrm{ng} \mathrm{ml}^{-1}\right)$. Patients with intact membranes will not have any IGFBP-1/AFP in the cervicovaginal secretions (11). The IGFBP-1/AFP had a falsepositive rate of $2.7 \%$ in present study. It is unclear what factors contribute to these false-positives. It is possible that there was a microleakage of the amniotic fluid in the vagina that could not be detected with a physical examination, bedside laboratories and ultrasonography ${ }^{(11)}$. AbuFaza et $\boldsymbol{a l l}^{(\mathbf{5})}$ compar Amnioquick duo+ with traditional clinical assessment (TCA) (nitrazin and ferning test) found Amnioquick duo+ more superior in diagnosing PROM but the difference was not statistically significant between the individual tests of TCA ${ }^{(5)}$. Our study, agree with Abdelazim et $\boldsymbol{a l}^{(\mathbf{1 3 )}}$ study which did not show significant differences in the diagnostic accuracy between Amnioquick duo+ and TCA. The sensitivity and specificity of the IGFBP-1/AFP test in this study was high agree with study done by Thomasino et al. (14) evaluated the diagnostic values for monoclonal/polyclonal immunoglobulin to detect IGFBP-1/AFP (ROM+). The sensitivity and specificity were $99 \%$ and $91 \%$, respectively, in the study by Thomasino et al. versus $100 \%$ and $97.3 \%$ in our current study. The performance of two rapid tests for the diagnosis of PROM based on the detection of IGFBP-1/AFP and placental alphamicroglobulin-1 (PAMG-1) in cervicovaginal secretions were compared by Eleje et al. (9) Sensitivity, specificity, and accuracy for Amnioquick duo+ were 97.9\%, 97.6\%, and 97.9\%, which were higher than the levels for PAMG-1, of $95.3 \%, 90.0 \%$, and $95.7 \%$, respectively (not significant). Accuracy of Amnioquick duo+ versus PAMG-1 in equivocal (pooling = negative) cases was $(98.4 \%$ vs $96.8 \%)$ at $\geq 34 \mathrm{GW}$ but each was $100.0 \%$ at <34 GW (not significant). Eleje et al. ${ }^{\text {(9) }}$ concluded that both amnioquick duo+ and PAMG1 have a comparatively high diagnostic accuracy in identifying women with PROM, with a concordance rate of $97.0 \%$. In this study the high sensitivity, specificity and accuracy of Amnioquick duo+ agree with Eleje $\boldsymbol{e t} \boldsymbol{a l} .{ }^{(7)}$ study in which two hundred and thirty-six women were recruited. Three women were excluded from the final analysis due to lack of follow-up data and failure to meet inclusion criteria. Two hundred and thirty- 
three women had complete data for analysis. The specificity and sensitivity values for TCA were $76.2 \%$ and $85.2 \%$, which were lower than those of Amnioquick duo+, which were $97.6 \%$ and $97.9 \%$, respectively. The accuracy of Amnioquick duo+ was statistically higher $97.9 \%$ vs. $83.7 \%$. In equivocal cases (pooling = negative), the accuracy of Amnioquick duo+ vs. TCA was $98.4 \%$ vs. $69.4 \%$ at $\geq 34$ weeks gestation and $100.0 \%$ vs. $71.4 \%$ at $<34$ weeks gestation. Eleje et al. $^{(7)}$ concluded the performance matrix of Amnioquick duo+ ${ }^{\circledR}$ was superior to that of TCA for diagnosing PROM even in equivocal cases. Our study high sensitivity, specificity and accuracy of Amnioquick duo+ higher than previous study done by Ruanphoo and Phupong ${ }^{(11)}$, in which one hundred patients were recruited. The mean gestational age was 37.6 weeks (range 25 to 41 weeks). Twentysix percent were preterm and $74 \%$ were at term. IGFBP-1/AFP immunoassay had a sensitivity of $94.1 \%$, specificity of $87.5 \%$, positive predictive value of $97.5 \%$, negative predictive value of $73.7 \%$ and accuracy of $93 \%$ in diagnosing ROM. Ruanphoo and Phupong ${ }^{(11)}$, concluded IGFBP1/AFP immunoassay is a rapid immunoassay test for diagnosing ROM with a high sensitivity and specificity. This test can be used as an alternative method for diagnosis of ROM.

\section{CONCLUSION}

This study concluded that IGFBP-1/AFP (AmnioQuick ${ }^{\circledR}$ Duo+) test is rapid, reliable, noninvasive, easy and accurate bedside immunoassay test, better than nitrazine test and can used as complementary test to improve the management of women with premature fetal membranes rupture.

\section{CONFLICTS OF INTEREST}

There are no conflicts of interest.

\section{REFERENCES}

1. Kim SA, Park KH and Lee SM (2016): Non-invasive prediction of histologic chorioamnionitis in women with preterm premature rupture of membranes. Yonsei. Med. J., 57:461-468.

2. Phupong $\mathbf{V}$ and Taneepanichskul $\mathbf{S}$ (2003): Prelabour rupture of membranes. J. Paediatr. Obstet. Gynaecol., 29:25-32.
3. Deckmyn B, Chieux V, Ammeux F et al. (2016): Comparison of immunochromatographic tests Actim( $(\circledR)$ Prom and Amnioquick(®) Duo for the rapid detection of premature rupture of membranes. Ann. Biol. Clin. (Paris),73(4):407-411.

4. Koike N, Oi H, Naruse $\mathrm{K}$ et al., (2016): Evaluation of vaginal fluid squamous cell carcinoma antigen test in diagnosis of premature rupture of membranes. J. Matern. Fetal Neonatal Med., 19:1-4.

5. AbuFaza ML, Abdelazim IA, AlSherbeeny MM et al. (2016): AmnioQuickR Duo+ for diagnosis of premature fetal membranes rupture. Int. J. Reprod. Contracept. Obstet. Gynecol., 5:674-679.

6. Liang DK, Qi HB, Luo X et al. (2016): Comparative study of placental $\alpha$ microglobulin-1, insulin-like growth factor binding protein-1 and nitrazine test to diagnose premature rupture of membranes: a randomized controlled trial. J. Obstet. Gynaecol. Res., 40(6):1555-1560.

7. Eleje GU, Ezugwu EC, Eke AC et al. (2016): Comparison of the duo of insulinlike growth factor binding protein-1 /alpha fetoprotein (Amnioquick duo+®) and traditional clinical assessment for diagnosing premature rupture of fetal membranes. J. Perinat. Med., 45(1):105-112.

8. Deering SH, Patel N, Spong CY et al. (2007): Fetal growth after preterm premature rupture of membranes: is it related to amniotic fluid volume? J. Matern. Fetal Neonatal Med., 20(5):397-400.

9. Eleje GU, Ezugwu EC, Eke AC et al. (2017): Accuracy and response time of dual biomarker model of insulin-like growth factor binding protein-1/ alpha fetoprotein (Amnioquick duot) in comparison to placental alpha-microglobulin-1 test in diagnosis of premature rupture of membranes. J. Obstet. Gynaecol. Res., 43(5):825-833.

10. Abdelazim IA and Makhlouf HH (2012): Placental alpha microglobulin-1 (AmniSure(®) test) for detection of premature rupture of fetal membranes. Arch. Gynecol. Obstet., 285(4):985-9. 
11. Ruanphoo $P$ and Phupong V (2015): Evaluation of the performance of the insulin-like growth factor-binding protein1/alpha-fetoprotein test in diagnosing ruptured fetal membranes in pregnant women. J Perinatol., 35(8):558-560.

12. Aboulenien W, Azzam A, Saleh $\mathrm{F}$ et al. (2009): Insulinlike growth factor binding protein-1 in cervico-vaginal secretions as an indicator of premature rupture of membranes: comparison with nitrazine test and amniotic fluid index. Int. J. Gynecol. Obstet., 107(2):413-729.
13. Abdelazim IA, Al-Sherbeeny MM, Ibrahim MEM et al. (2016): Insulin-like growth factor binding protein-1/alphafetoprotein versus placental alpha microglobulin-1 for diagnosis of premature fetal membranes rupture. Acta. Medica. International,3:69-74.

14. Thomasino $T$, Levi $C$, Draper $M$ et al. (2013): Diagnosing rupture of membranes using combination monoclonal/polyclonal immunologic protein detection. J. Reprod. Med., 58:187-194. 